\title{
Mapping road crossings for visually impaired people
}

\author{
Yuhao Jiang ${ }^{\mathrm{a}, *}$, María-Jesús Lobo ${ }^{\mathrm{a}}$, Sidonie Christophe ${ }^{\mathrm{a}}$, Christophe Jouffrais ${ }^{\mathrm{b}}$ \\ ${ }^{a}$ LASTIG, Univ Gustave Eiffel, ENSG, IGN, F-94160 Saint-Mande, France, yuhao.jiang@ensg.eu, Maria-Jesus.Lobo@ign.fr, \\ Sidonie.Christophe@ign.fr \\ ${ }^{b}$ IRIT, University of Toulouse, France, christophe.jouffrais@irit.fr \\ * Corresponding author
}

Keywords: tactile map, visual impairment, accessibility, orientation and mobility

\begin{abstract}
:
Tactile maps are an important source of information to communicate spatial knowledge and facilitate the mobility of people with visual impairment (PVIs) (Espinosa et al., 1998). With map representations that correspond well to the important environmental features perceived by the PVIs (Lobben \& Lawrence, 2012), potentially enhanced with audio and interactions (Brock et al., 2015), the maps can efficiently assist them to acquire the necessary spatial knowledge for their journey. But the maps are often not available for road crossings, especially complex ones with traffic islands, which are particularly challenging in the journeys of PVIs. Most existing mobility-related automated tactile mapping tools work on the scale of neighborhoods with more emphasis on road networks and building footprints (Minatani et al., 2010; Štampach \& Mulíčková, 2016; Touya et al., 2019), and even when it's possible to zoom in to a road crossing level (Červenka et al., 2016), there is little detailed information about the layout of the road crossings regarding e.g. traffic islands or pedestrian crossings that are critical to the execution of a safe cross (Wiener et al., 2010). These road crossing maps are still being hand-made by professionals in a tailored and time-consuming manner.
\end{abstract}

To better facilitate PVIs mobility around the road crossings with (semi-)automated mapping, we still need to better understand how spatial knowledge could be efficiently communicated through non-visual manners in this particular situation, including how the crossing is perceived, mentally represented, and related with the tactile representations. In the framework of ANR ActivMAP project, inspired by the practice in handmade tactile mobility maps, we aim to explore the design possibilities for tactile maps for road crossing and produce them in a (semi-)automated manner in an experimental approach. Our short-term objective is to produce a simple map with less complex geometries while still presenting the necessary information related to a road crossing to facilitate the PVIs' understanding of its layout.

We conducted the experiment with OpenStreetMap data, following the tactile graphic guidelines (e.g. The Braille Authority of North America 2010). Considering the potential use case of the map (portable), the scale of the map is initially set at 1:1000, intended to be printed on microcapsule paper of A4 or A5 size. Based on available handmade maps made by professional tactile document makers and orientation and mobility instructors, we initially identified the objects to be included in the map as streets, traffic islands, pedestrian crossings, building blocks, and sidewalks. The prototype pipeline of our experiment follows a classical cartographic pipeline in terms of geometry processing and styling. The geometry processing mainly consists of the transformation and generalization of the geometries existing in the dataset (displacement and transformation of the streets, generalization of building blocks, transformation of pedestrian crossing points), and the estimations of object geometries that are not present in the datasets (e.g. traffic islands and sidewalks). Since there is no standard regarding tactile symbolizations for objects on the road crossing level, the last step of our pipeline consists of exploring multiple possible styling options, as observed from various handmade maps. The pipeline is implemented in QGIS.

To illustrate our proposal, an example result of a road crossing map derived from the pipeline is shown in Figure 1. This road crossing was chosen based on the available handmade maps we had from a professional tactile document maker (Matin, 2018). The styling choice for this example is based on the styles used in handmade maps, taking into consideration the tactile graphic guidelines (solid lines for street boundaries, dashed lines for pedestrian crossings, and two types of textures for the area features). The difference between the handmade map and the pipeline output is mainly due to the elimination of (minor) streets and the inclusion of a vegetation patch by the tactile document maker.

Other styling options could be further explored: with different graphic representation options applied, all inspired by styling examples from existing handmade maps. For the same set of objects, such different representations could convey different emphasis, with various richness of information and levels of complexity that can potentially impact the understanding of the map by the PVIs. Figure 2 shows three styling options as examples of a sub-area of Figure 1: traffic islands that are split (a potential cue for PVIs) might be emphasized with a distinctive texture or with slightly modified geometries that better resembles the actual ground situation (a); buildings and sidewalks can be represented 
simultaneously with different textures to distinguish (b); pedestrian accessible areas (traffic islands, sidewalks) can be rendered with the same texture to convey the "walkability" of the area (c).

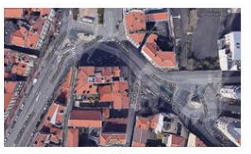

a)

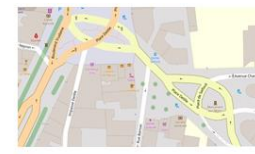

b)

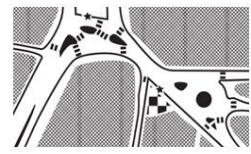

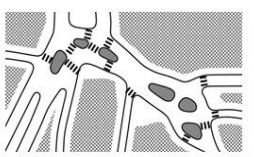

d)

Figure 1 Example image and maps of a complex road crossing. a) the aerial image of the crossing (Google Map); b) OpenStreetMap data with their typical style; c) the handmade map; d) a resulting tactile map for PVIs we (semi)automatically made in QGIS.
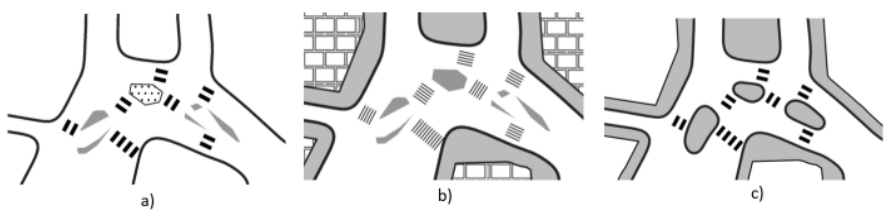

Figure 2 Different styling options: a): only traffic islands with distinctive textures and detailed geometry; with buildings and sidewalk information omitted; b) traffic islands with distinction in geometry but the same texture, distinct building and sidewalk styling; c) sidewalk and island taking the same texture to convey the "walkability" of the area.

The experiment will serve as a starting point to further explore design options for tactile road crossing maps. This process could also be extended to incorporate other objects such as tram lines and parking lots, and be further adapted to other complex situations such as roundabouts and multi-level crossings involving bridges and tunnels. In the ongoing work of an upcoming user study, the resulting maps as well as other possible design options are going to be evaluated with final users and mobility instructors.

\section{Acknowledgements}

This study is funded by the French Research Agency ANR, in the framework of ACTIVMAP ANR-19-CE19-0005 (Computer Assisted Map Design for Visually Impaired People) project (2020-2024).

\section{References}

Brock, A. M., Truillet, P., Oriola, B., Picard, D., \& Jouffrais, C. (2015). Interactivity Improves Usability of Geographic Maps for Visually Impaired People. Human-Computer Interaction, 30(2), 156-194. https://doi.org/10.1080/07370024.2014.924412

Červenka, P., Břinda, K., Hanousková, M., Hofman, P., \& Seifert, R. (2016). Blind friendly maps: Tactile maps for the blind as a part of the public map portal (Mapy.cz). Lecture Notes in Computer Science (Including Subseries Lecture Notes in Artificial Intelligence and Lecture Notes in Bioinformatics), 9759, 131-138. https://doi.org/10.1007/978-3-31941267-2_18

Espinosa, M. A., Ungar, S., Ochaita, E., Blades, M., \& Spencer, C. (1998). Comparing methods for introducing blind and visually impaired people to unfamiliar urban environments. Journal of Environmental Psychology, 18, $277-287$.

Lobben, A., \& Lawrence, M. (2012). The Use of Environmental Features on Tactile Maps by Navigators Who Are Blind. Professional Geographer, 64(1), 95-108. https://doi.org/10.1080/00330124.2011.595619

Martin, D. (2018). Rapport D’activités: Comment réaliser un plan de ville accessible à des déficients visuels? [Report of Activities: How to make a city map accessible to the visually impaired?]. Clermont-Ferrand

Minatani, K., Watanabe, T., Yamaguchi, T., Watanabe, K., Akiyama, J., Miyagi, M., \& Oouchi, S. (2010). Tactile map automated creation system to enhance the mobility of blind persons - Its design concept and evaluation through experiment. Lecture Notes in Computer Science (Including Subseries Lecture Notes in Artificial Intelligence and Lecture Notes in Bioinformatics), 6180 LNCS(PART 2), 534-540. https://doi.org/10.1007/978-3-642-14100-3_80

Štampach, R., \& Mulíčková, E. (2016). Automated generation of tactile maps. Journal of Maps, 12, 532-540. https://doi.org/10.1080/17445647.2016.1196622

The Braille Authority of North America. (2010). Guidelines and Standards for Tactile Graphics. http://www.brailleauthority.org/tg/web-manual/index.html

Touya, G., Christophe, S., Favreau, J., Ben, A., Touya, G., Christophe, S., Favreau, J., Ben, A., Automatic, R., Touya, G., \& Christophe, S. (2019). Automatic derivation of on-demand tactile maps for visually impaired people: first experiments and research agenda. International Journal of Cartography, 5(1), 67-91. https://doi.org/10.1080/23729333.2018.1486784

Wiener, W. R., Welsh, R. L., \& Blasch, B. B. (2010). Foundations of Orientation and Mobility, Volume II, Instructional Strategies and Practical Applications (Third Edit). American Foundation for the Blind. 